\title{
The time of embryonic axis formation in quail eggs
}

\author{
Md. Anisur Rahman ${ }^{1}$ and Norio Yoshizaki ${ }^{2}$ \\ ${ }^{1}$ Department of Zoology, University of Rajshahi, Bangladesh. E-mail: ar_zool.uk@fastermail.com \\ ${ }^{2}$ Department of Animal Science, Faculty of Applied Biological Sciences, Gifu University, Japan.
}

The chalazae constitute an axis of rotation around which the egg can rotate, maintaining the germ upward within the inner liquid albumen (Burley \& Vadehra, 1989; Rahman et al., 2007, 2009). When the outer part of the egg envelope, the eggshell, rotates in the uterus, the egg yolk remains nearly motionless, and the chalazae become coiled (Clavert, 1962). As a result of this rotation, the blastoderm is forced into an oblique position, and the embryo develops an antero-posterior axis with its head in the direction of rotation (Kochav \& EyalGiladi, 1971). Vintemberger \& Clavert (1960) showed that most eggs rotate in the uterus with their sharp end toward the cloaca, in which case the embryo develops according to Von Baer's rule; its tail develops toward the observer holding the sharp end towards the right of the observer. The present study aimed to find out the time of embryonic axis formation by incubating oviductal eggs of quail.

Japanese quail (Coturnix japonica) of the wild-type strain were reared individually in cages in a poultry house with $16 \mathrm{~h}$ illumination per day. They were fed a layer diet ad libitum and killed by cervical dislocation, which was approved by an Institutional Animal Care and Use Committee of Gifu University (No. 06120). The oviductal eggs were removed from 20-week-old females at an appropriate time after oviposition.

Collected eggs were positioned horizontally with the equatorial side up, marked at the top of the eggshell, and incubated at $39^{\circ} \mathrm{C}$ and at $60 \%$ humidity until Day 3 of incubation, at which time the embryonic axis appears. After incubation, the eggshell was cut around the mark, inverted, and the orientation of the embryonic head was determined, with the orientation of naturally spawned eggs being $0^{\circ}$ according to the rule of Von Baer (Fig.1). If the anterior direction shifted toward the blunt end, the value is expressed with a plus sign, whereas if it shifted toward the sharp end, it is shown with a minus sign.

Ovulated eggs were located in the oviductal uterus during 5-25 $\mathrm{h}$ after the previous oviposition in Japanese quail (Iwasawa et al., 2010; Rahman et al., 2009; Woodard and Mather, 1964). Overall, 114 oviductal eggs were examined. Among them, 26 eggs were collected from females during $5-17 \mathrm{~h}$ after the previous oviposition, but incubation of these eggs resulted in arrested development at the blastoderm embryo stage, with no axis formation (data not shown).
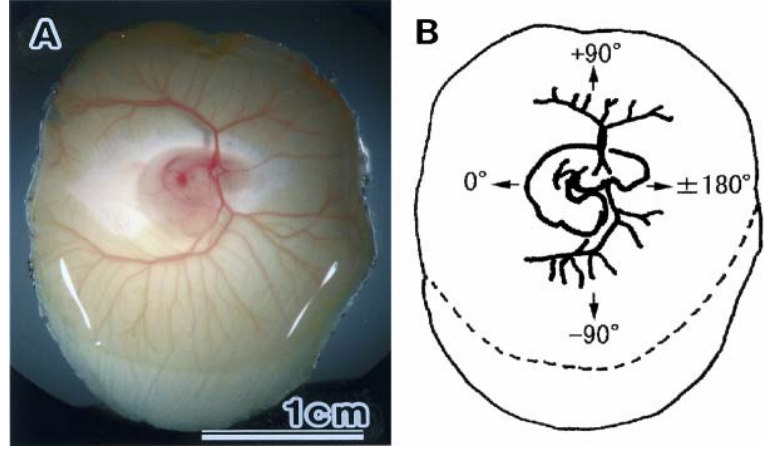

Fig. 1 Micro-photograph of oviductal egg incubated for 3 days $(A)$ and its illustration (B), showing the definition of axis orientation with the anterior direction of embryo being $0^{\circ}$, according to the rule of Von Baer. The blunt end of the egg is at the top. View from inside.

Forty-four eggs collected during 17-25 h developed an embryonic axis (Fig. 2A). Of these, the direction of the antero-posterior axis of the embryo in the eggs collected during 17-21 $\mathrm{h}$ deviated widely from the fundamental position determined by the rule of Von Baer (see, Clavert, 1962; Kochav and Eyal-Giladi, 1971), in a random direction. On the other hand, eggs collected during 21-25 $\mathrm{h}$ developed an embryonic axis converging into the fundamental position.

In the remaining 44 eggs collected during 17-25 h, development was arrested at the thin blastoderm with occasional blood cells, and the embryonic axis was not formed (Fig. 2B). Such arrested development was mostly observed in eggs collected during 17-21 h. Since the location of these blastoderms was just beneath the mark made on the top of eggshells and since the location of embryonic bodies, if developed, always shifted from the mark, some shift of the blastoderm from the top position may happen in the latter but not in the former. This means that embryonic development does not proceed unless its axial formation occurs. The reason for the developmental arrest observed in eggs collected during 5-17 $\mathrm{h}$ is unknown. 

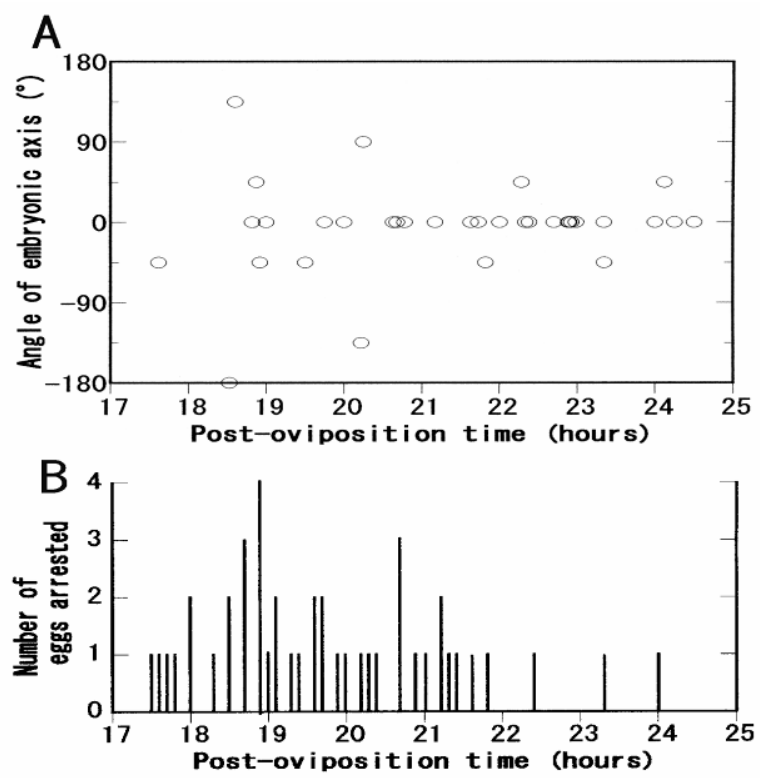

Fig. 2 Angle of the anterior direction of embryonic axis that developed in eggs collected from oviducts at various hours after the previous oviposition $(A)$ and the number of eggs in which development arrested (B).

Eggshells and the cuticle layer are formed in the uterus. The matrix of eggshell is produced by granular cells of mucosal epithelia and calcified between 7 and $20 \mathrm{~h}$ after the previous oviposition (Iwasawa et al., 2010). The cuticle materials were produced by ciliated cells of mucosal epithelia between 21-25 h (Rahman et al., 2009). The appearance of $10-\mu \mathrm{m}$-wide posts on the surface of the mucosal epithilia during eggshell formation and their disappearance during cuticle formation was interesting (Iwasawa et al., 2010). Retreat of the posts leads to the formation of air canals in the eggshell, but it also means that the egg in the uterus is immobile from $7 \mathrm{~h}$ to $21 \mathrm{~h}$ and becomes mobile after $21 \mathrm{~h}$ (Rahman et al., 2009). This transition may be triggered by progesterone, which stimulates the release of the resident sperm from the sperm storage tubules in the utero-vaginal junction (Ito et al., 2011) with the same time schedule as that of cuticle formation.

It is said that the peristalsis of the uterus wall rotates the shell materials slowly in one direction while the yolk tends to return the egg to a vertical position, keeping the blastoderm tilted obliquely through the commitment of chalazae (Gerhart and Kirschner, 1997). Kochav \& Eyal-Giladi (1971) experimentally showed that the embryo head pointed in the direction of rotation. The present study showed that the embryonic anterio-posterior axis is formed during 21-25 $\mathrm{h}$ after the previous oviposition with an aid of cuticle material as a lubricant for egg rotation; however, the molecular mechanism of axis formation is still unknown. Such a study may be possible in the future using eggs collected during $17-21 \mathrm{~h}$, since they are competent for axis formation but this has not been determined yet.

The present findings disclose the determination of the time of antero-posterior axis formation in embryos of the Japanese quail (Coturnix japonica). Oviductal eggs from the females during 5-25 $\mathrm{h}$ after the previous oviposition incubated for several days in a horizontal position with the equatorial side up. Eggs during 5-17 $\mathrm{h}$ after the previous oviposition showed arrested development at the blastoderm embryo stage, with no axis formation. The direction of the embryonic axis in the eggs collected during 17-21 $\mathrm{h}$ showed a random orientation or developmental arrest. But the eggs collected during 21-25 $\mathrm{h}$ showed the development of the embryonic axis converging into the fundamental position determined by the rule of Von Baer. These results indicate that the embryonic antero-posterior axis is formed during 21-25 $\mathrm{h}$ after the previous oviposition in quail.

\section{References}

Burley, R.W. \& Vadehra, D.V. 1989. The Avian Egg: Chemistry and Biology. John Wiley \& Sons, New York

Clavert, J. 1962. Symmetrization of the egg of vertebrates. Advan Morphoge. 2: 27-60

Gerhart, J. \& Kirschner, M. 1997. Cells, Embryos, and Evolution. Blackwell Science, Malden, Massachusetts.

Ito, T., Yoshizaki, N., Tokumoto, T., Ono, H., Yoshimura, T., Tsukada, A., Kansaku, N., \& Sasanami, T. 2011. Progesterone is a sperm-releasing factor from the spermstorage tubules in birds. Endocri. 152: 3952-3962

Iwasawa, A., Rahman, M.A., Roy, T.K., Moriyama, A. \& Yoshizaki, N. 2010. Morphological and histochemical changes in the uterus epithelium during eggshell formation in quail. J Poult Sci. 47: 183-189

Kochav, S. \& Eyal-Giladi, H. 1971. Bilateral symmetry in chick embryo determination by gravity. Science. 171: 1027-1029

Rahman, M.A., Baoyindeligeer., Iwasawa, A., Yoshizaki, N. 2007. Mechanism of chalaza formation in quail eggs. Cell Tissue Res. 330: 535-543

Rahman, M.A., Moriyama, A., Iwasawa, A. \& Yoshizaki, N. 2009. VMO-II mediates the binding of the chalaziferous layer with the vitelline membrane in quail eggs. J Poult Sci. 46: $240-248$

Vintemberger, P. \& Clavert, J. 1960. Sur le déterminisme de la symétrie bilatérale chez les Oiseaux. XIII. C R Soc Biol. 154: 1072-1076

Woodard, A. E. \& Mather, F.B. 1964. The timing of ovulation, movement of the ovum through the oviduct, pigmentation and shell deposition in Japanese quail (Coturnix coturnix japonica). Poult Sci. 43: 1427-1432 